\title{
Endovascular treatment for aortic arch pathologies: chimney, on-the-table fenestration, and in-situ fenestration techniques
}

\author{
Chang Shu ${ }^{1,2 \#}$, Bowen Fan ${ }^{1 \#}$, Mingyao Luo ${ }^{1}$, Quanming $\mathrm{Li}^{2}, \mathrm{Kun} \mathrm{Fang}{ }^{1}, \mathrm{Ming} \mathrm{Li}^{2}, \mathrm{Xin}^{\mathrm{Li}^{2}}$, Hao $\mathrm{He}^{2}$, \\ Tun Wang ${ }^{2}$, Chenzi Yang ${ }^{2}$, Yunfei Xue ${ }^{1}$, Haoyu Gao ${ }^{1}$, Jiawei Zhao ${ }^{1}$ \\ ${ }^{1}$ State Key Laboratory of Cardiovascular Disease, Fuwai Hospital, National Center for Cardiovascular Diseases, Chinese Academy of Medical \\ Sciences \& Peking Union Medical College, Beijing 100037, China; ${ }^{2}$ Department of Vascular Surgery, Second Xiangya Hospital, Central South \\ University, Changsha 410011, China \\ Contributions: (I) Conception and design: C Shu, B Fan; (II) Administrative support: C Shu; (III) Provision of study materials or patients: C Shu, B \\ Fan, M Luo, Q Li, K Fang, M Li, X Li, H He, T Wang, C Yang; (IV) Collection and assembly of data: B Fan, M Luo, Q Li, K Fang, M Li, X Li, \\ H He, T Wang, C Yang, Y Xue, H Gao, J Zhao; (V) Data analysis and interpretation: C Shu, B Fan, M Luo, K Fang; (VI) Manuscript writing: All \\ authors; (VII) Final approval of manuscript: All authors. \\ \#These authors contributed equally to this work. \\ Correspondence to: Chang Shu. Fuwai Hospital, National Center for Cardiovascular Diseases, No 167 Beilishi Road, Beijing 100037, China. Email: \\ Changshu_vascular@163.com; changshu@vip.126.com.
}

Background: Revascularization of the supra-aortic major branches in thoracic endovascular aortic repair (TEVAR) is challenging owing to the complex anatomic configuration of aortic arch pathologies. This study aims to evaluate the feasibility, effectiveness, and safety of three major techniques—chimney, fenestrated, and in-situ fenestration — for patients with aortic arch pathologies.

Methods: A retrospective analysis was performed involving 234 patients with aortic arch lesions, who underwent TEVAR with adaptations in technique (chimney, fenestrated, or in-situ fenestration) between January 2016 and December 2017.

Results: One hundred and twenty-six patients underwent the chimney technique (98 single chimneys, 24 double chimneys, and four triple chimneys); one hundred and two patients (102/234) were treated with on-the-table fenestration technique (92 single fenestrations, nine double fenestrations, and one double fenestration plus innominate artery chimney); and the remaining six patients underwent in-situ needle fenestration technique. Overall, indications included aortic dissections (99/234), aortic arch aneurysms (60/234), penetrating aortic ulcers (72/234), and re-interventions (3/234). The technical success rates were $99.6 \%$. There were five cases of early all-cause mortality. The patency rates of overall branches were $99.6 \%$. There were 15 cases with type Ia endoleak-14 in the chimney group (11.1\%) and one in the on-the-table fenestration group (1\%). Five patients underwent re-interventions. The median follow-up time for all patients was 28 (range, 16-41) months.

Conclusions: Our experience suggests that chimney, on-the-table fenestration, and in-situ needle fenestration techniques are feasible, effective, and safe treatment options for aortic arch pathologies with encouraging mid-term results. Long-term durability concerns require further evaluation.

Keywords: Endovascular procedure; aortic diseases; aneurysm; dissecting

Submitted Dec 02, 2019. Accepted for publication Feb 05, 2020.

doi: $10.21037 /$ jtd.2020.03.10

View this article at: http://dx.doi.org/10.21037/jtd.2020.03.10 


\section{Introduction}

Treating aortic arch pathologies such as aortic dissections and aneurysms is challenging for vascular surgeons. Although conventional surgical repair remains the therapeutic gold standard, surgical repair with cardiopulmonary bypass and deep hypothermic circulatory arrest is associated with a high risk of mortality (1). The hybrid technique is the refined alternative to open surgery; however, it is still associated with high mortality (2). High-risk patients with comorbidities are deemed unsuitable to receive surgical repairs.

Thoracic endovascular aortic repair (TEVAR) has seen rapid development and application in the past two decades. Owing to the development of stent-grafts and endovascular techniques, the indications for TEVAR have expanded, and it is currently used to treat aortic arch pathologies. Despite favourable long-term results, the applicability of custommade stent-grafts is limited because of technical difficulties and long manufacturing delays, especially in urgent cases with severe acute end-organ ischemia or impending rupture (3). Moreover, custom-made stent-grafts are not commercially available at all times in many institutes.

The chimney (C-TEVAR), on-the-table fenestration (F-TEVAR), and in-situ fenestration (I-TEVAR) are TEVAR-assistive techniques, that could be accomplished with readily available devices. C-TEVAR has earned worldwide popularity owing to the procedure being less complicated, feasible across various anatomic features, and being applicable in a bail-out situation (4). F-TEVAR, also known as physician-modified fenestrated graft technique, is a quick procedure for an off-the-shelf stent-graft by the surgeon in the operating room (5-7). I-TEVAR, including in-situ laser fenestration and in-situ needle fenestration, is also a potential technique for revascularization of supraaortic branches $(8,9)$. Because each of these techniques has strengths and limitations, endovascular treatment strategies for aortic arch diseases are still controversial. Herein, we provided the outcomes of 234 consecutive patients who received C-TEVAR, F-TEVAR, or I-TEVAR for aortic arch pathologies, aimed to assess the feasibility, effectiveness, and safety of these techniques and probably provide good alternatives for open repair in selected patients.

\section{Methods}

The patient cohort was studied retrospectively from
January 1, 2016 to December 31, 2017. The institutional review boards of both Fuwai Hospital, National Centre for Cardiovascular diseases and the Second Xiangya Hospital approved the study, and the need for informed consent was waived (ID: D171100002917004, Dated: January 1, 2016).

\section{Patient eligibility}

An interdisciplinary board composed of endovascular and cardiovascular surgeons, radiologists, and anaesthesiologists was responsible for comprehensively evaluating each patient to decide treatment options. Patients who met the following criteria were considered suitable candidates for undergoing assistive techniques in TEVAR: those with (I) aortic arch pathologies involving single or multiple supraaortic branches; (II) aortic arch pathologies including aortic dissection (AD), penetrating aortic ulcer (PAU), and pseudoaneurysm of aortic arch (PAA); and (III) patients who were aged with comorbidities and hence unsuitable for the risks of open or hybrid surgery.

The exclusion criteria for endovascular treatment were as follows: patients with (I) proximal intimal tear of the $\mathrm{AD}$ involving the ascending aorta; (II) concomitant pathologies such as cardiac tamponade and severe aortic regurgitation which necessitated surgical treatment; and (III) other anatomical formations not suitable for TEVAR, such as severe tortuousness in the access route arteries, angulation or compression, or very large diameter of the maximum trans-aortic diameter severely limiting device use.

The most suited TEVAR-assistive technique was selected based on the following principles: F-TEVAR was the first option when aortic arch pathologies were located merely at the inner curve of the aortic arch, because a large-sized fenestration could be made to efficiently align the fenestration with the vascular branches. When pathologies involved the outer curve of the aortic arch and the diameters of the branch artery were large, F-TEVAR with a small-sized fenestration was used. Varieties of fenestrations are shown in Figure 1. When the angulation between the aortic arch and the descending aorta was hostile for orientation of the fenestration (Figure 2), and when branch artery dissection was present, C-TEVAR was employed. When the proximal intimal tear in the $\mathrm{AD}$ was located at the outer curve and very close to the branch arteries, the risk of an endoleak was considerable with the previous two techniques. In such cases, I-TEVAR was used. 

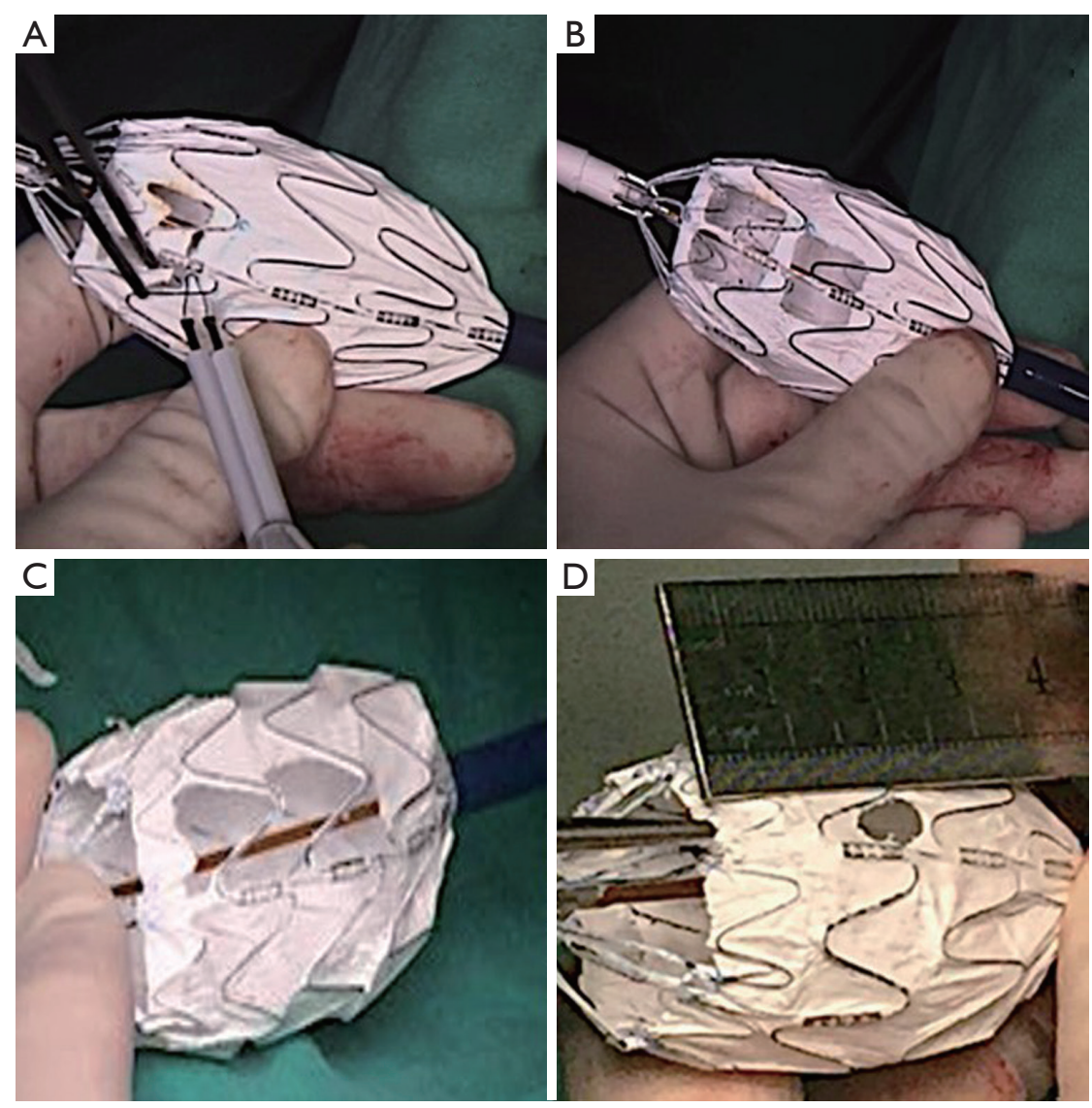

Figure 1 Fenestrations modified by surgeons. (A) The Ankura thoracic stent-graft was partially unsheathed, measured, and fashioned using a laser scalpel alongside the spiral support strut of the aortic stent-graft; (B) a double fenestration was created for both the LSA and LCCA; (C) a large fenestration was made for preserving the left subclavian artery (LSA); (D) a small fenestration was made to align with the LSA to prevent from endoleaks.

\section{Operative techniques}

\section{Pre-procedure preparation}

All patients were administered general anaesthesia in our hybrid operating suite. The common femoral artery was exposed surgically or percutaneously using Perclose ProGlide suture device (Abbott Laboratories Co., Ltd, USA).

\section{Selection for stent-graft}

In the C-TEVAR group, Valiant (Medtronic, Inc, Minneapolis, MN, USA) and Hercules (MicroPort Medical Co., Ltd, Shanghai, China) were selected as the main thoracic aortic stent-grafts, because these grafts have better flexibility than Ankura (Lifetech, Scientific Co., Ltd, Shenzhen, China) for stent-graft apposition with branch endografts. In contrast, Ankura stent-graft was used in F-TEVAR, because the unique marker in an Ankura graft could facilitate stent-graft orientation. The proximal part of the Ankura stent-graft has two differently shaped radiopaque markers-an " 8 "-shaped marker on one side and an O-shaped marker on the opposite side. Moreover, Ankura also contains a spiral support strut, which is aligned with the " 8 "-shaped marker. The linear alignment of the " 8 "-shaped marker and the spiral strut of Ankura stentgraft indicated the exact position of the fenestration, which helped surgeons to accurately orient the fenestration to the orifice of the aortic branches. In contrast, Valiant stentgraft has four almost identical markers that need further modification to distinguish one marker for the orientation procedure.

Two types of covered endografts, Fluency (C.R. Bard, Inc, Murray Hill, NJ, USA) and Viabahn (Gore \& Associates, Flagstaff, AZ, USA), were mostly used as grafts 

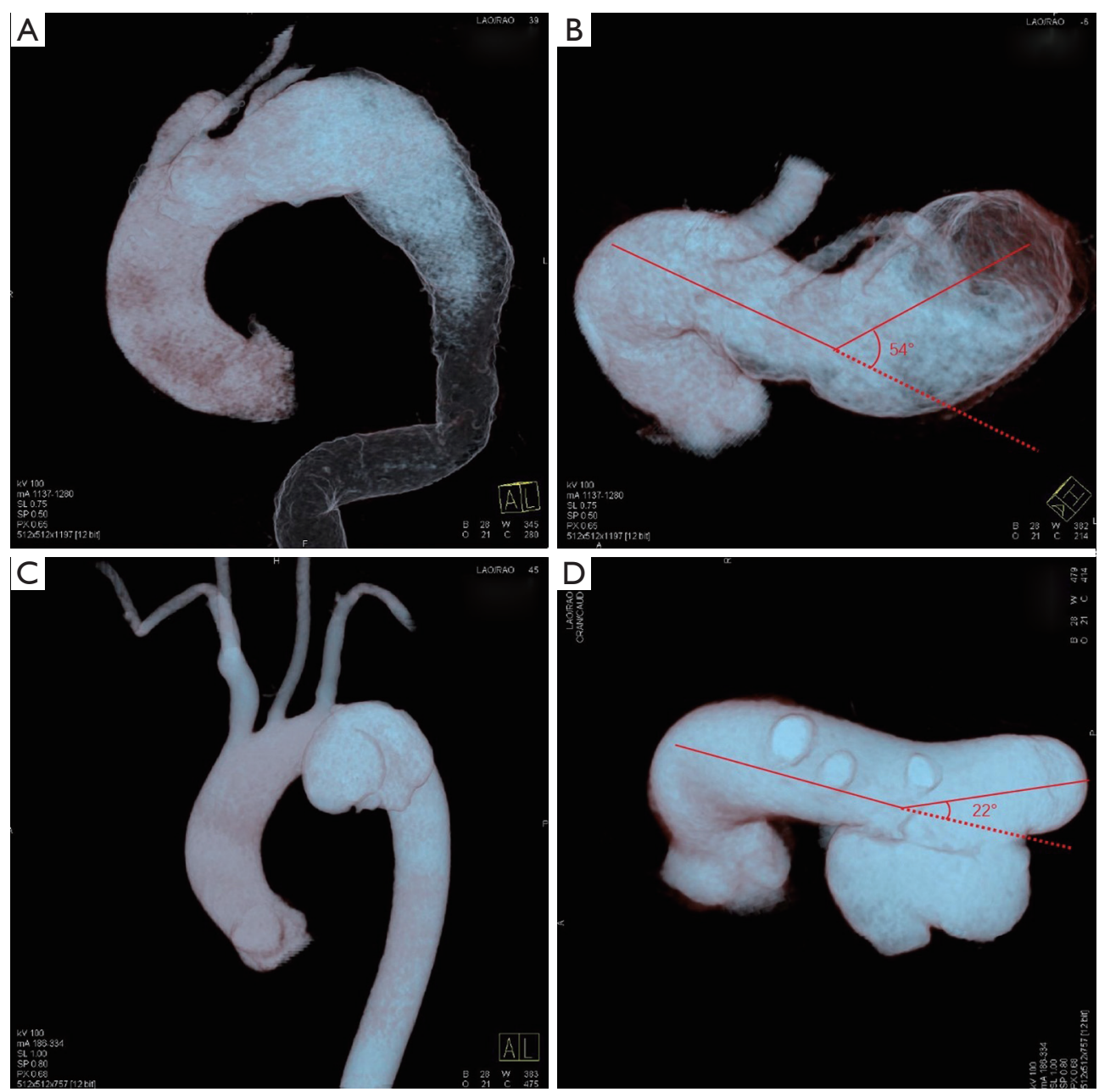

Figure 2 Morphology of the aortic arch. (A,B) The vertical and horizontal 3D fusion image of a patient with aortic arch aneurysm. Red lines showed an angle of 54 degrees between the aortic arch and the descending aorta. For this hostile aortic arch angulation, chimney technique would be selected. (C,D) The vertical and horizontal 3D fusion image of another patient with aortic arch aneurysm. Red lines showed an angle of 22 degrees. This less angulated aortic arch allows planning of on-the-table fenestration technique.

for branch arteries. A bare endograft (Biotronik Medical Co., Ltd, Berlin, Germany) was also used in the F-TEVAR group.

\section{Chimney technique procedure}

Our standardized C-TEVAR procedure has been previously described $(10,11)$. Basically, the chimney graft for the supraaortic branches was inserted via brachial/carotid access and the aortic stent-graft was introduced through the common femoral access. Then, the chimney grafts were released parallel to the main stent-graft with the proximal segment protruding at least $2.0 \mathrm{~cm}$ into the aortic lumen and the distal end remaining in the branch artery, while ensuring that at least $1.0 \mathrm{~cm}$ of overlapping segment remained between the two grafts. The aortic stent-grafts were selected with a $0-20 \%$ oversizing, and the chimney graft was selected with a $0-5 \%$ oversizing. Routine moulding of the chimney graft was performed via a balloon.

\section{On-the-table fenestration procedure}

Adequate understanding of the anatomical structure via the pre-operative CTA was essential for the success of orientation. Digital subtraction angiography (DSA) was performed and the diameters of vascular branches were measured to design a fenestration with comparable size to the target vessels. The proximal part of the aortic stent-graft 

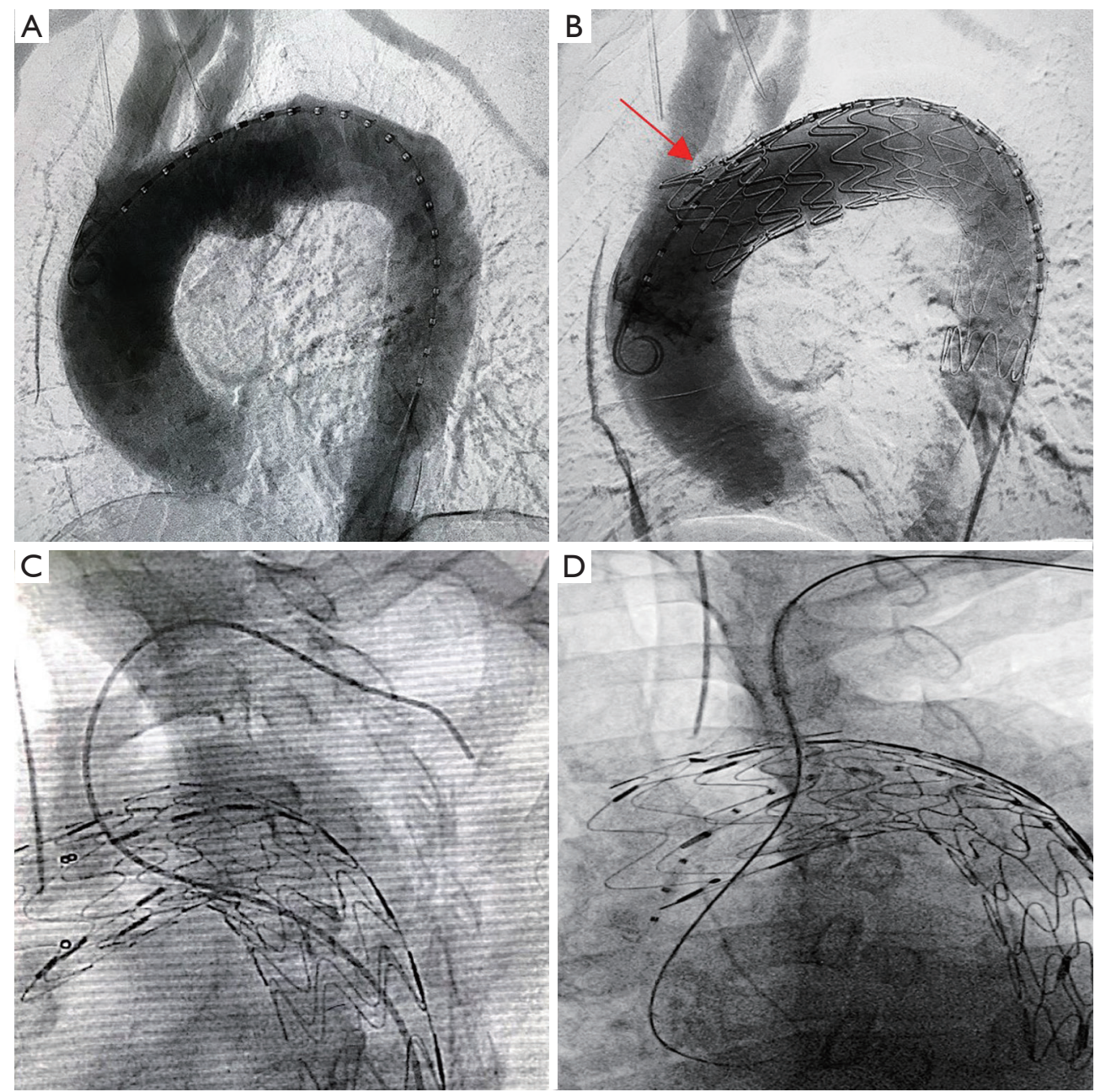

Figure 3 Procedures of on-the-table fenestration technique. (A) DSA showed a penetrating aortic ulcer (PAU) involving both the LSA and LCCA. (B) The fenestrated stent-graft was oriented towards both the LSA and LCCA, ensuring the eight-shaped radiopaque marker (red arrow) and the spiral support strut align with two target vessels. Completion DSA showed isolation of the PAU while patency of all branches. (C) Antegrade route for endografts delivery from the common femoral artery. (D) Retrograde route for endografts delivery from the brachial artery.

was unsheathed in vitro, measured, and marked by a sterile pen. Then, the fenestration was fashioned by a scalpel alongside the spiral support strut beside the eight-shaped marker (Figure 1A). The edges of the fenestration were kept neat, and all fragments were carefully removed (Figure 1B). Then, the fenestrated stent-graft was carefully reassembled. Guided by an extra-stiff guide wire, the stent-graft was inserted into the descending aorta via femoral access. After confirming that the " 8 "-shaped marker and the spiral strut of the stent-graft were aligned with the target vessel, the stent-graft was carefully released. Then, angiography was performed to check the results (Figure $3 A, B$ ).
The indications for stenting of aortic branches, already preserved by F-TEVAR, were as follows: (I) the IA and LCCA, being crucial brain supplying arteries, should always be stented by endografts, and (II) the following evaluation results showed that the LSA was not well preserved and required stenting: (i) intraoperative surveillance of bilateral blood pressure showed a significant blood pressure difference between bilateral brachial arteries; (ii) completion DSA showed poor blood flow through the orifice of the LSA. Either antegrade femoral access (Figure 3C) or retrograde brachial/carotid access (Figure $3 D$ ) could be used for delivery of endografts into the aortic branches. Femoral 

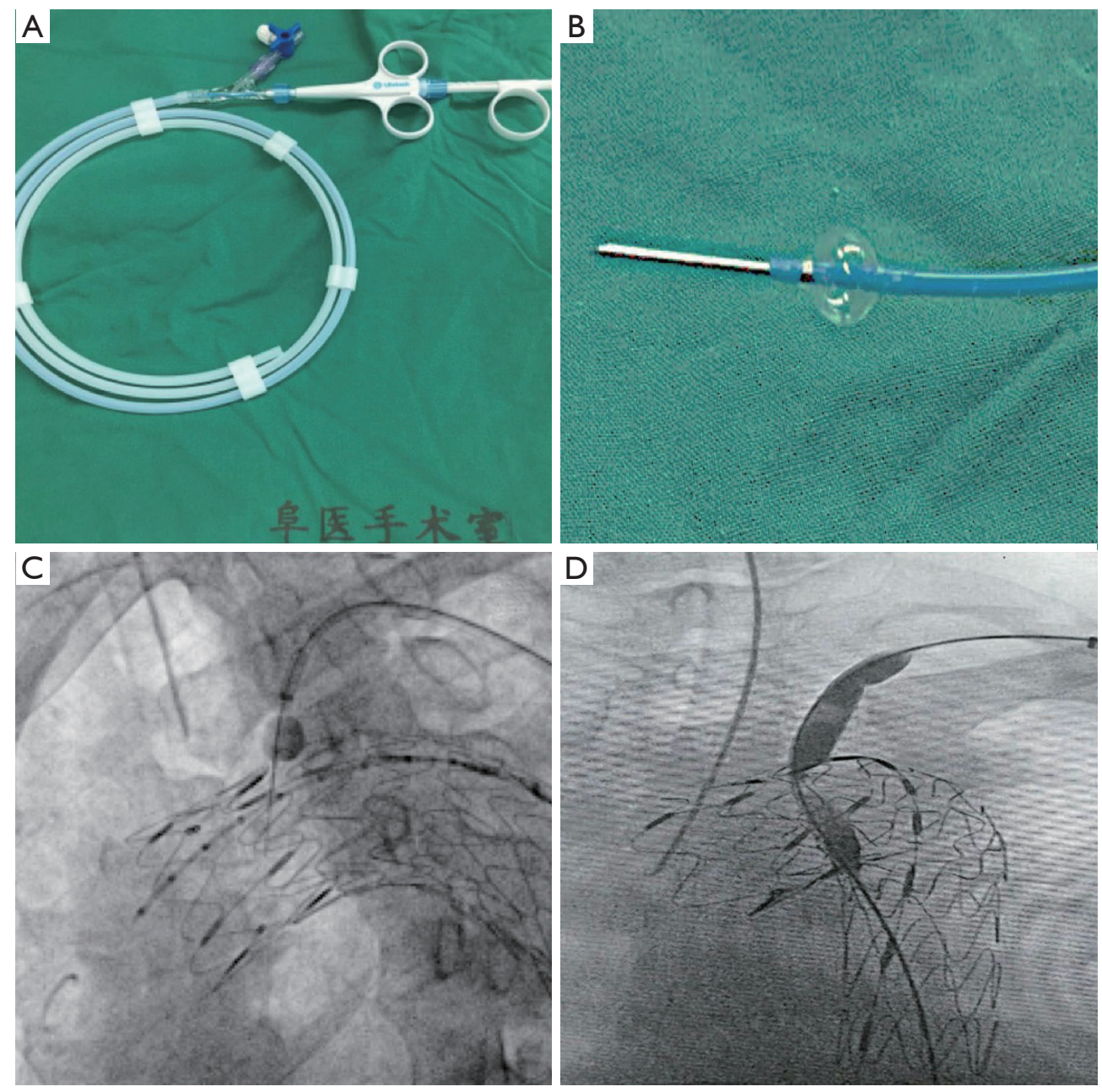

Figure 4 In-situ needle fenestration technique. (A) The balloon expandable puncture needle. (B) The puncture needle activates when inflating the balloon. (C) An in-situ fenestration was created by the puncture needle, and a wire was advanced to the aortic lumen. (D) A balloon catheter of comparable size was used to expand the fenestration.

access required no extra brachial or carotid incisions, and was generally tried first.

\section{In-situ needle fenestration procedure}

First, the aortic stent-graft was introduced to the aortic arch and deployed at the planned landing zone. Then, a balloon expandable puncture needle (Lifetech, Scientific Co., Ltd, Shenzhen, China; Figure $4 A, B$ ) was introduced through the Fustar steerable vascular sheath (Lifetech) from the brachial access and advanced to the orifice of the LSA. The fenestration was created by inflating the balloon to activate the puncture needle (Figure $4 C$ ). Then, the guidewire was passed through the newly created fenestration to reach the aortic lumen. After balloon expansion of the fenestration site (Figure 4D), a covered stent-graft was deployed to ensure patency of the aortic branch. Finally, the stent-graft for the aortic branch was again dilated via a balloon, and completion DSA was performed to check the result.

\section{Post-operative management}

Each patient was managed postoperatively by oral medication including antiplatelet drugs, antihypertensive drugs, and beta-blockers. The follow-up for each patient was scheduled at $1,3,6$, and 12 months, and annually thereafter.

\section{Statistical analysis}

Continuous data are expressed as the mean $( \pm \mathrm{SD})$ or 
median (range). Cumulative survival was analysed using Kaplan-Meier survival curves. Analyses were conducted using SPSS 23.0 software (IBM Corporation, Somers, NY, USA). GraphPad Prism 8 (GraphPad Software, San Diego, CA, USA) was used for graphical representation of data.

\section{Results}

From January 1, 2016 to December 31, 2017, a total of 859 patients underwent surgeries for aortic arch pathologies in both Fuwai Hospital and Second Xiangya Hospital. We excluded 424 patients undergoing open arch replacement and 201 patients receiving the hybrid technique. Finally, 234 patients who received TEVAR combined with assistive techniques were included. Of these, $126(53.8 \%)$ in the C-TEVAR group underwent the chimney technique, 102 $(43.6 \%)$ in the F-TEVAR group underwent on-the-table fenestration technique, and $6(2.6 \%)$ in the I-TEVAR group underwent the in-situ needle fenestration technique. Baseline characteristics and patient demographics of each group are detailed in Table 1.

In the C-TEVAR group, 95, 28, and three cases of singlechimney, double-chimney, and triple-chimney techniques were performed. A total of 158 chimney grafts (CGs) were utilised, including 100 Fluency grafts (LSA, n=60; LCCA, $\mathrm{n}=31 ; \mathrm{IA}, \mathrm{n}=9$ ) and 58 Viabahn grafts (LCCA, n=14; LSA, $\mathrm{n}=43$; ARSA, $\mathrm{n}=1$ ). In the F-TEVAR group, 91 single fenestrations (90 for the LSA, 1 for the ARSA), nine double fenestrations (7 for LSA and LCCA, 2 for LSA and aberrant left vertebral artery), and one double fenestration for LCCA and LSA combined with chimney for IA were performed. Thirteen branch arteries were stented (IA, $\mathrm{n}=1$; LCCA, $\mathrm{n}=2$, LSA, $\mathrm{n}=10$ ) using 12 covered endografts (Fluency, $\mathrm{n}=1$; Viabahn, $\mathrm{n}=11$ ) and 1 bare endograft (Biotronik, $\mathrm{n}=1$ ). Three (23.1\%) LSA stent-grafts were deployed by femoral access, while the remaining 10 stent-grafts were deployed from brachial or carotid access. In the I-TEVAR group, all six patients underwent the in-situ fenestration technique for LSA. More details are listed in Table 2.

\section{Periprocedural outcomes}

Technical success was defined as isolation of pathologies while ensuring patency of the supra-aortic branches in the completion DSA. This was achieved in 233 of 234 patients (99.6\%). Alignment of fenestration to the LSA failed in one patient in the F-TEVAR group $(1 / 102,1.0 \%)$ because of the lack of experience at the start of our practice. The occluded
LSA in this case was revascularized by stenting the bail-out chimney graft and remained patent during the follow-up.

In all, 5 of 234 (2.1\%) patients died within 30 days. One patient who received C-TEVAR for a huge aortic arch pseudoaneurysm died of sudden cardiac arrest 15 days postoperatively. Another patient with $\mathrm{AD}$ developed renal ischemic complications and died of renal failure. The third patient with $\mathrm{AD}$ died of bowel ischemia necrosis because of severely compressed distal aortic true lumen, despite emergent measures taken to expand the true lumen. In the F-TEVAR group, one patient died of ischemic stroke and the other died from bowel ischemia necrosis.

Early type Ia endoleak, detected exclusively by the postoperative CTA on discharge, was found in 15 patients $(6.4 \%)-14(11.1 \%)$ in the C-TEVAR group and one $(1.0 \%)$ in the F-TEVAR group. The median ICU stay duration was 1 (range, 0-8) day in the C-TEVAR group, 1 (range, $0-7$ ) day in the F-TEVAR group, and 1 (range, 1-2) day in the I-TEVAR group, and the respective median hospital stays were 6 (range, 1-22) days, 6 (range, 5-19) days, and 6 (range, 5-8) days.

\section{Mid-term follow-up outcomes}

The LSA of one patient in the F-TEVAR group was found occluded at the 12-month follow-up. The LSA was not stented because intraoperative evaluation of the LSA after deployment of fenestrated stent-graft showed favourable results. The CTA showed that the occlusion was near the orifice of the LSA, which was not caused by stent-graft migration.

Post-operative outcomes are listed in Table 3, and Kaplan-Meier estimates of mortality are shown in Figure 5. Ischemic stroke was diagnosed based on symptoms and images. Overall, 4 (1.7\%) patients developed stroke-2 $(1.6 \%)$ in the C-TEVAR group and $2(2.0 \%)$ in the F-TEVAR group. No patient had paraplegia. The median follow-up time for all patients was 28 (range, 16-41) months.

The 15 patients who developed type Ia endoleaks were managed by modifications to oral medication, i.e., by stopping antiplatelet drugs and increasing antihypertensive drugs. Two patients with endoleak in the C-TEVAR group who had refractory chest pain and increasing aortic diameters received re-interventions. The first patient, who underwent the chimney technique (Medtronic 34-34-200 mm and Fluency $8-80 \mathrm{~mm}$ for a $30-\mathrm{mm}$ diameter landing zone and a 7-mm diameter LSA), received angioplasty for the chimney endograft one year later, which significantly 
Table 1 Baseline characteristics of patients

\begin{tabular}{|c|c|c|c|}
\hline Characters & On-the-table fenestration $(n=102)$ & Chimney technique $(n=126)$ & In situ fenestration $(n=6)$ \\
\hline Age, mean (SD) & $59.1(10.9)$ & $58.0(13.5)$ & $55(4.9)$ \\
\hline \multicolumn{4}{|l|}{ Timing of treatment } \\
\hline Elective & $60(58.8)$ & $56(44.4)$ & $3(50.0)$ \\
\hline Rupture & $3(7.1)$ & $5(7.1)$ & $0(0)$ \\
\hline Visceral ischemia & $15(35.7)$ & $24(34.3)$ & $1(33.3)$ \\
\hline Limb ischemia & $11(26.2)$ & $21(30.0)$ & $2(66.7)$ \\
\hline Renal ischemia & $10(23.8)$ & $16(22.9)$ & $0(0)$ \\
\hline \multicolumn{4}{|l|}{ Indication for treatment } \\
\hline Aortic dissection & $37(36.3)$ & $56(44.4)$ & $6(100.0)$ \\
\hline Chronic aortic dissection & $3(2.9)$ & $3(2.4)$ & $0(0)$ \\
\hline Thoracic aortic aneurysm & $14(13.7)$ & $21(16.7)$ & $0(0)$ \\
\hline Penetrating aortic ulcer & $42(41.2)$ & $30(23.8)$ & $0(0)$ \\
\hline Aortic pseudoaneurysm & $8(7.8)$ & $17(13.5)$ & $0(0)$ \\
\hline Type la endoleak & $1(1.0)$ & $2(1.6)$ & $0(0)$ \\
\hline Intramural hematoma & $18(17.6)$ & $16(12.7)$ & $0(0)$ \\
\hline Heart failure & $5(4.9)$ & $7(5.6)$ & $0(0)$ \\
\hline COPD & $9(8.8)$ & $9(7.1)$ & $0(0)$ \\
\hline Previous stroke & $9(8.8)$ & $12(9.5)$ & $0(0)$ \\
\hline Traumatic aortic injury & $2(2.0)$ & $3(2.4)$ & $0(0)$ \\
\hline Marfan syndrome & $1(1.0)$ & $2(1.6)$ & $0(0)$ \\
\hline
\end{tabular}

Values are $\mathrm{n}(\%)$ unless age are mean (SD). COPD, chronic obstructive pulmonary diseases. *Multiple organ ischemia: ischemia of two or more vital organ systems.

reduced the endoleak. The other patient with endoleak received a chimney endograft (Fluency $100-60 \mathrm{~mm}$ ) poorly attached to the vessel wall of the LSA $(10 \mathrm{~mm})$ because of insufficient oversizing. One year later, another endograft was placed distal to the previous chimney endograft, and the endoleak stopped after balloon moulding for both grafts.
Three other patients, including two with retrograde type A aortic dissection (RAAD) and one with compressed IA endograft, received re-interventions. One patient with $\mathrm{AD}$ and intramural hematoma involving the aortic arch presented with severe acute renal ischemia, and thus underwent emergency C-TEVAR with Medtronic 38-38- 
Table 2 Procedural findings

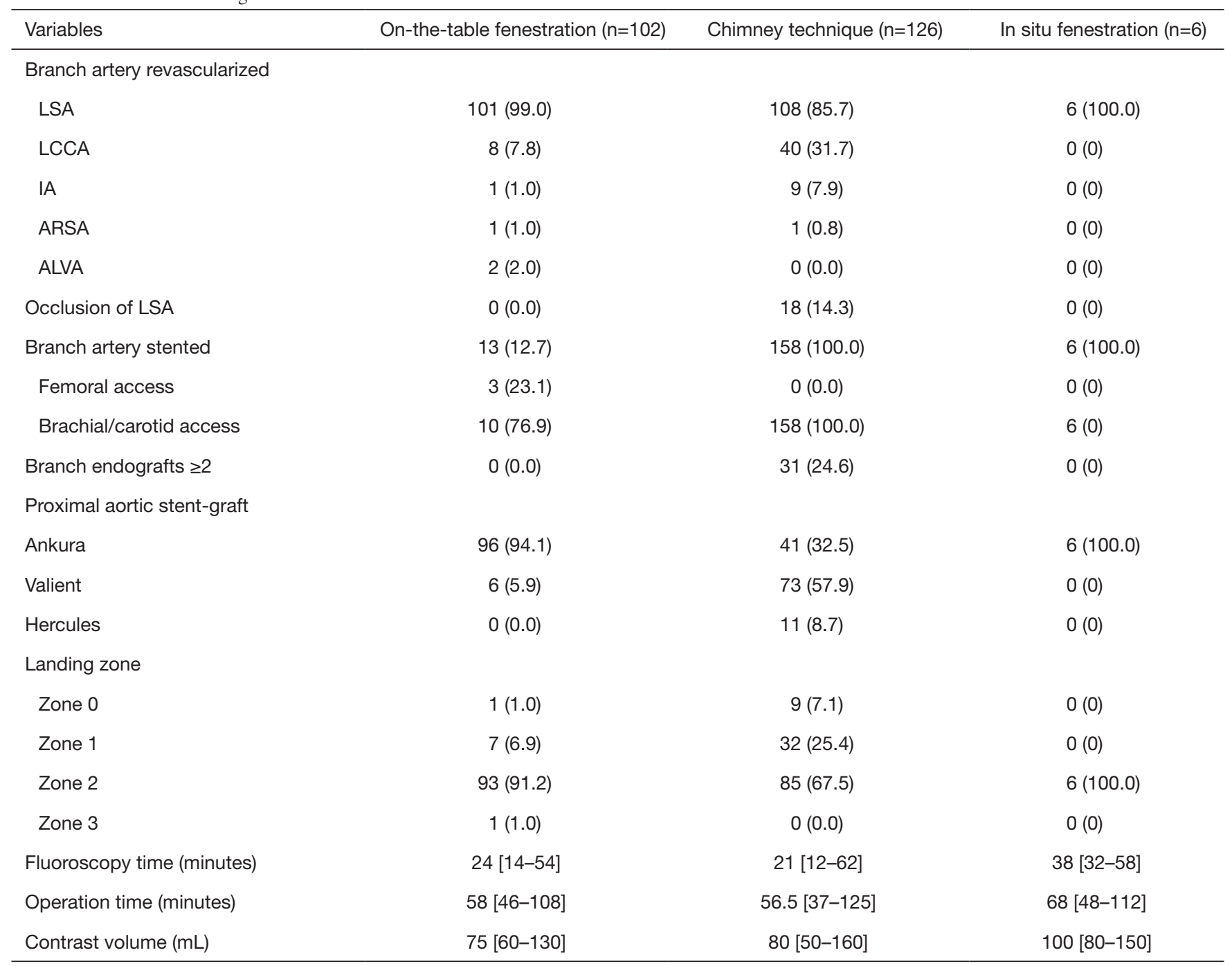

Values are $\mathrm{n}(\%)$ except that procedural time, operation time and contrast volume are median [range]. LSA, left subclavian artery; LCCA, left common carotid artery; IA, innominate artery; ARSA, aberrant subclavian artery; ALVA, aberrant left vertebral artery; Fluoroscopy time, duration between the first angiography and the completion angiography; Operation time, time from the beginning of surgical cutdown to the completion of the operation.

$200 \mathrm{~mm}$ and Fluency $8-80 \mathrm{~mm}$. The other patient who developed refractory chest pains was found to have RAAD at the 3-month follow-up and received open surgery after 19 months. Another patient was diagnosed with intramural hematoma that progressed to a PAU with refractory pain after 2 weeks. Therefore, the patient was treated by C-TEVAR, with Ankura 36-32-160 mm and Fluency 6-60 mm; however, CTA showed the presence of RAAD one month later. This patient underwent open heart surgery after 7 months. The patient with a compressed endograft of the IA received balloon moulding 1 year later. The above five patients recovered uneventfully after re-interventions.

\section{Discussion}

Our experience with TEVAR-chimney, on-the-table fenestration, and in-situ fenestration techniques-for aortic arch pathologies showed encouraging outcomes with low mortality and complication rates, indicating that these endovascular techniques were safe and feasible options. Patients recovered well and quickly and benefited from lessinvasive treatments. 
Table 3 Postoperative outcomes

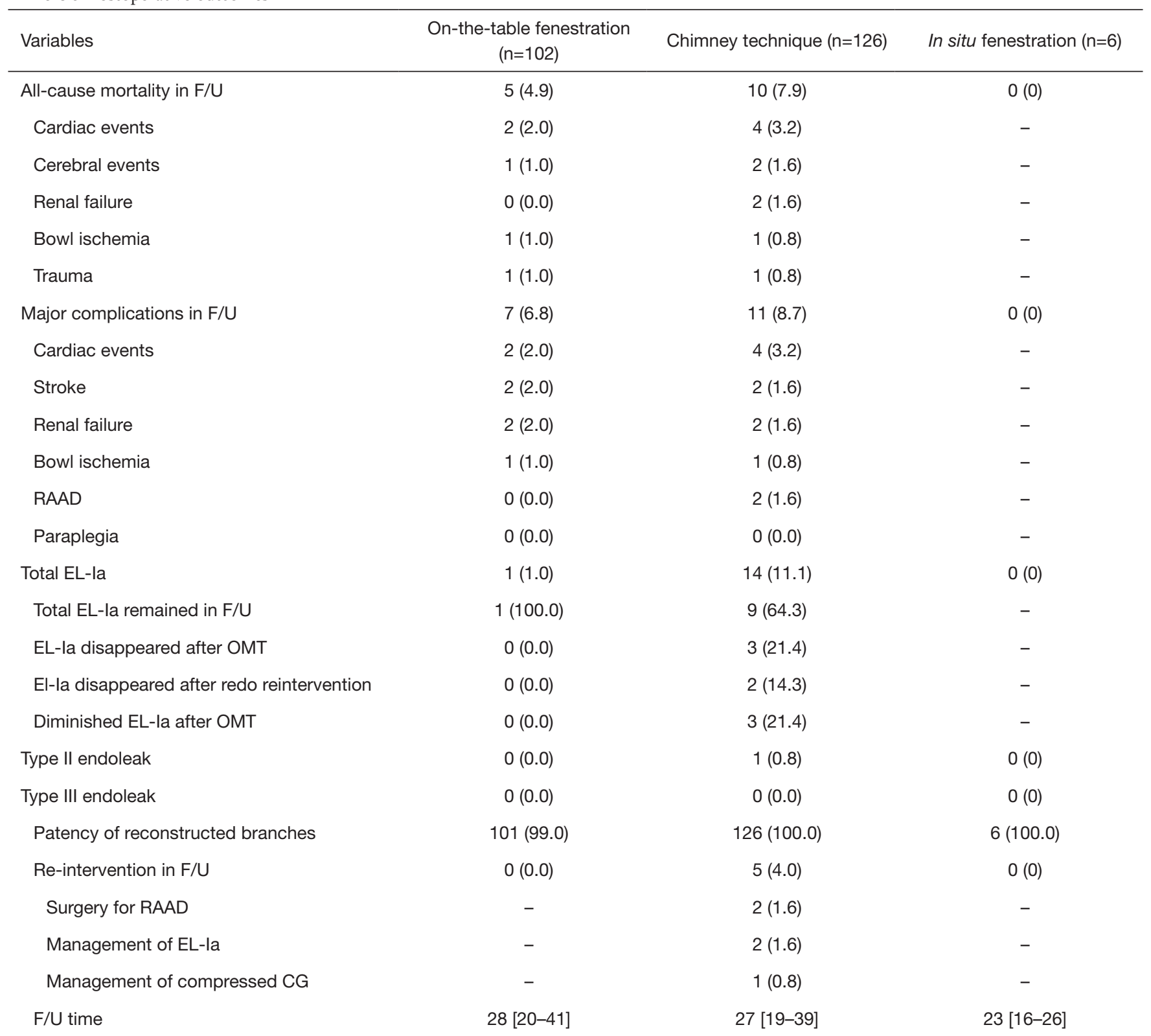

Values are $\mathrm{n}(\%)$ except that time are median months [range]. F/U, follow-up; RAAD, retrograde type A aortic dissection; EL-la, type la endoleak; OMT, oral medicine treatment; CG, chimney graft.

In our practice, each technique has its strengths and limitations. C-TEVAR is a simple procedure that could adapt to the complex aortic arch anatomy, such as severe tortuosity of the aortic arch and angulation of the supraaortic branches. Moreover, the chimney technique could be used in bail-out situations like unintentional coverage of branch vessels (12). However, the main limitation of this approach is the susceptibility of endoleaks, because of the so-called 'gutter' between stent-grafts. In the present study, $11 \%$ patients in the C-TEVAR group developed endoleaks, which was consistent with recent publications that reported 9.9-14.3\% and $13.0-19.7 \%$ endoleak rates in the single and double chimney techniques, respectively $(10,11,13-15)$. Moreover, chimney endografts are permanently compressed by the thoracic stent-graft which presents a potential threat to the long-term stability and patency of the graft. 


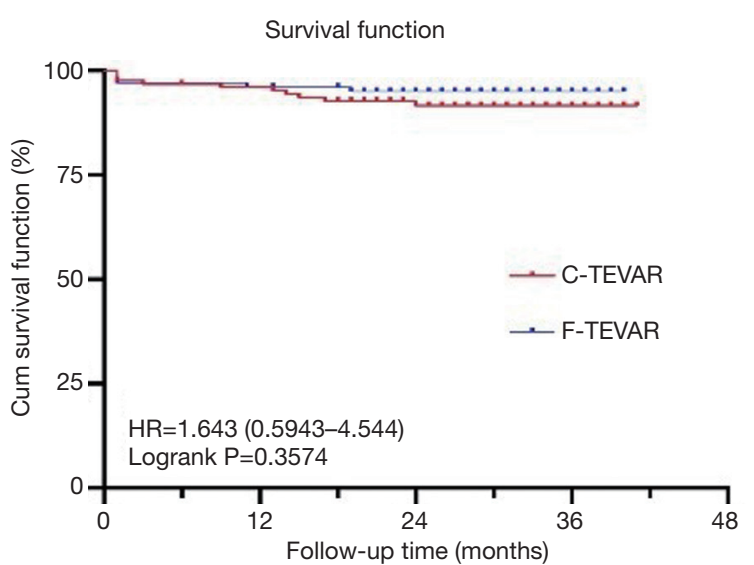

Number at risk

$\begin{array}{lrrrr}\text { C-TEVAR: } & 126 & 120 & 84 & 23 \\ \text { F-TEVAR: } & 102 & 98 & 67 & 20\end{array}$

Figure 5 Kaplan-Meier estimates of overall survival.

F-TEVAR is a promising and more judicious technique used to repair the aorta without altering the anatomic structures. In our practice, we took advantage of the characteristics of Ankura stent-grafts, thus making the design, modification, and orientation procedures much more efficient and effective. In the present study, only one of $102(1.0 \%)$ patients developed an endoleak, which was consistent with recent publications that reported endoleak rates of $0-4.2 \%(5-7)$. These results might be attributed to our strategy of choosing F-TEVAR more often in cases of aortic arch pathologies that involved only the inner curve of the aorta, as noted in the $41.2 \%$ patients treated for penetrating ulcers.

I-TEVAR has shown great potential in endovascular treatment for aortic arch pathologies $(9,16)$. Both C-TEVAR and F-TEVAR are susceptible to endoleaks when the proximal intimal tear of the $\mathrm{AD}$ is very close to the branch arteries. I-TEVAR, with a relatively smaller fenestration, may prevent endoleak occurrence. Although the sample size of the I-TEVAR group $(\mathrm{n}=6)$ was rather limited, all six patients experienced technical success without endoleaks. However, the I-TEVAR approach had several limitations. First, I-TEVAR required specific anatomic conditions, namely a vertical angle of the target branch to the aortic arch. Tortuosity and sharp angle of the branch vessels could greatly increase the procedural difficulty (9). Second, temporary carotid arteries coverage raises concerns for ischemic complications; hence, I-TEVAR was not the first choice for revascularization of the LCA and IA. Third, I-TEVAR was associated with greater procedural and fluoroscopy times and volume of contrast owing to more procedural steps and technical difficulties than C-TEVAR and F-TEVAR (17).

Therefore, based on the individual strengths and limitations and encouraging outcomes of these three techniques in the early and mid-term period, each of these three techniques has application in endovascular surgery. However, long-term results remain uncertain and need further validation. In future, we hope to report another study of these techniques in a larger cohort and with a longer follow-up period.

\section{Conclusions}

Total endovascular treatments for patients with aortic arch pathologies are challenging because of the diverse vascular anatomies of both the aorta and the aortic branches. The chimney, fenestrated, and in-situ needle fenestration techniques in TEVAR provided encouraging mid-term results in our selected patient cohort and are hence feasible, effective, and offer a safe alternative to open surgery. Additional long-term studies are required to evaluate the robustness and applicability of these techniques.

\section{Acknowledgments}

Funding: National Natural Science Foundation, China (No. 81870345); Beijing Municipal Science \& Technology commission (No. D171100002917004); Grant of CAMS Initiative for Innovative Medicine, China (NO. 2016-I2M1-016).

\section{Footnote}

Conflicts of Interest: The authors have no conflicts of interest to declare.

Ethical Statement: The authors are accountable for all aspects of the work in ensuring that questions related to the accuracy or integrity of any part of the work are appropriately investigated and resolved. The institutional review boards of both Fuwai Hospital, National Centre for Cardiovascular diseases and the Second Xiangya Hospital approved the study, and the need for informed consent was waived (ID: D171100002917004, Dated: January 1, 2016).

Open Access Statement: This is an Open Access article 
distributed in accordance with the Creative Commons Attribution-NonCommercial-NoDerivs 4.0 International License (CC BY-NC-ND 4.0), which permits the noncommercial replication and distribution of the article with the strict proviso that no changes or edits are made and the original work is properly cited (including links to both the formal publication through the relevant DOI and the license). See: https://creativecommons.org/licenses/by-nc$\mathrm{nd} / 4.0 \%$.

\section{References}

1. De Rango P, C Ferrer, C Coscarella, et al. Contemporary comparison of aortic arch repair by endovascular and open surgical reconstructions. J Vasc Surg 2015;61:339-46.

2. Moulakakis KG, Mylonas SN, Markatis F, et al. A systematic review and meta-analysis of hybrid aortic arch replacement. Ann Cardiothorac Surg 2013;2:247-60.

3. Mastracci TM, Eagleton MJ, Kuramochi Y, et al. Twelveyear results of fenestrated endografts for juxtarenal and group IV thoracoabdominal aneurysms. J Vasc Surg 2015;61:355-64.

4. Lindblad B, Bin Jabr A, Holst J, et al. Chimney Grafts in Aortic Stent Grafting: Hazardous or Useful Technique? Systematic Review of Current Data. Eur J Vasc Endovasc Surg 2015;50:722-31.

5. Canaud L, Baba T, Gandet T, et al. Physician-Modified Thoracic Stent-Grafts for the Treatment of Aortic Arch Lesions. J Endovasc Ther 2017;24:542-48.

6. Canaud L, Morishita K, Gandet T, et al. Homemade fenestrated stent-graft for thoracic endovascular aortic repair of zone 2 aortic lesions. J Thorac Cardiovasc Surg 2018;155:488-93.

7. Zhu J, Zhao L, Dai X, et al. Fenestrated Thoracic Endovascular Aortic Repair Using Physician Modified Stent Grafts for Acute Type B Aortic Dissection with Unfavourable Landing Zone. Eur J Vasc Endovasc Surg 2018;55:170-76.

Cite this article as: Shu C, Fan B, Luo M, Li Q, Fang K, Li M, Li X, He H, Wang T, Yang C, Xue Y, Gao H, Zhao J. Endovascular treatment for aortic arch pathologies: chimney, on-the-table fenestration, and in-situ fenestration techniques. J Thorac Dis 2020;12(4):1437-1448. doi: 10.21037/ jtd.2020.03.10
8. Sonesson B, Dias N, Resch T, et al. Laser Generated In situ Fenestrations in Dacron Stent Grafts. Eur J Vasc Endovasc Surg 2016;51:499-503.

9. Katada Y, Kondo S, Tsuboi E, et al. Endovascular Total Arch Repair Using In Situ Fenestration for Arch Aneurysm and Chronic Type A Dissection. Ann Thorac Surg 2016;101:625-30.

10. Wang T, Shu C, Li M, et al. Thoracic Endovascular Aortic Repair With Single/Double Chimney Technique for Aortic Arch Pathologies. J Endovasc Ther 2017;24:383-93.

11. Wang T, Shu C, Li QM, et al. First experience with the double chimney technique in the treatment of aortic arch diseases. J Vasc Surg 2017;66:1018-27.

12. Derbel B, Tolenaar JL, Trimarchi S. Use of Chimney graft after accidental coverage of the left common carotid artery in TEVAR procedure. Tunis Med 2014;92:756.

13. Baldwin ZK, Chuter TA, Hiramoto JS, et al. Doublebarrel technique for endovascular exclusion of an aortic arch aneurysm without sternotomy. J Endovasc Ther 2008;15:161-5.

14. Bosiers MJ, Donas KP, Mangialardi N, et al. European Multicenter Registry for the Performance of the Chimney/Snorkel Technique in the Treatment of Aortic Arch Pathologic Conditions. Ann Thorac Surg 2016;101:2224-30.

15. Xue Y, Sun L, Zheng J, et al. The chimney technique for preserving the left subclavian artery in thoracic endovascular aortic repair. Eur J Cardiothorac Surg 2015;47:623-9.

16. Shang T, Tian L, Li D, et al. Favourable Outcomes of Endovascular Total Aortic Arch Repair Via Needle Based In Situ Fenestration at a Mean Follow-Up of 5.4 Months. Eur J Vasc Endovasc Surg 2018;55:369-76.

17. XiaoHui M, Li W, Wei G, et al. Comparison of supraarch in situ fenestration and chimney techniques for aortic dissection involving the left subclavian artery. Vascular 2019;27:153-160. 